\title{
Chain Retraction in Highly Entangled Stretched Polymer Melts
}

\author{
Hsiao-Ping Hsu* and Kurt Kremeit \\ Max-Planck-Institut für Polymerforschung, Ackermannweg 10, 55128, Mainz, Germany
}

\begin{abstract}
We use computer simulations to study the relaxation of strongly deformed highly entangled polymer melts in the nonlinear viscoelastic regime, focusing on anisotropic chain conformations after isochoric elongation. The Doi-Edwards tube model and its Graham-Likhtman-McLeish-Milner (GLaMM) extension, incorporating contour length fluctuation and convective constraint release, predict a retraction of the polymer chain extension in all directions, setting in immediately after deformation. This prediction has been challenged by experiment, simulation, and other theoretical studies, questioning the general validity of the tube concept. For very long chains we observe the initial contraction of the chain extension parallel and perpendicular to the stretching direction. However, the effect is significantly weaker than predicted by the GLaMM model. We also show that the first anisotropic term of an expansion of the 2D scattering function qualitatively agrees to predictions of the GLaMM model, providing an option for direct experimental tests.
\end{abstract}

PACS numbers: $83.80 \mathrm{Sg}, 83.50 .-\mathrm{v}$, 83.10.Rs

The reptation model and its extensions, based on conformational properties and entanglement effects in dense polymer systems, represent the basis of our current understanding of viscoelastic properties of modern polymer materials, which are omnipresent in our daily life products and in technology. In the linear viscoelastic regime, the theory originally developed by Doi, Edwards, and de Gennes [1 6] based on the original tube concept of Edwards [7] successfully describes dynamics and viscoelasticity, e.g., stress relaxation, of highly entangled polymer melts. It is strongly supported in detail by simulation [8-18] and experiment [19 22]. To account for finite chain length corrections, refinements of the original concept have been developed, namely the effect of contour length fluctuation (CLF) [4, 23 27], and constraint release (CR) 24, 28 33]. These modify pure reptation and correctly reproduce the disentanglement time of $\tau_{d, N} \propto N^{3.4}, N$ being the degree of polymerization of the chains. In the nonlinear viscoelastic regime, Doi and Edwards [5] assume that polymer chains in a melt deform affinely along the chain contour, following the sample deformation. On scales above the tube diameter this has recently been confirmed by us 34]. The chain radius of gyration along the stretching direction increases and simultaneously decreases in the perpendicular direction. Immediately after deformation - still within the affinely deformed tube - the stress along the contour of the chain causes an initial retraction along the tube. All linear dimensions of deformed chains are expected to first decrease, while the chains try to retract back into the tube. Following the refined Graham-Likhtman-McLeish-Milner (GLaMM) tube model 35] [includes CLF, CR, and convective constraint release $(\mathrm{CCR})]$ this initial retraction is expected to last for up to the Rouse time of the chains. However, it is not clear in which way these concepts apply to local conformational properties and the nonlinear viscoelasticity of polymer melts.

Based on neutron scattering experiments of highly stretched polystyrene melts Wang et al. 36] question the validity of the whole tube concept. By careful analysis of two-dimensional anisotropic small-angle neutron scattering (SANS) spectra of polymer melts having $Z=N / N_{e}=34$ entanglements per chain $\left(N_{e}\right.$ being the entanglement length), they could not observe the predicted initial chain retraction. Their subsequent molecular dynamics simulation [37] of a standard, fully flexible bead spring model of polymer melts [9, 12] of $Z=33$ supports their experimental findings (taking $N_{e} \approx 85$, as estimated through a primitive path analysis [38, 39], $Z \approx 24$ ). In contrast, earlier work on nonlinear rheology of highly entangled polymer melts [40 42] supports the theoretical prediction of chain retraction by SANS. In Refs [40, 42 44] the authors observe clear signatures of anisotropically deformed conformations of monodisperse entangled polystyrene melts in nonlinear flow, and even for unentangled chains subject to extremely fast flow [45]. In Refs. [40, 42] the authors also show the subsequent relaxation in agreement with the GLaMM tube model. In Ref. [41], Blanchard et al. observe a minimum in the deformed radius of gyration perpendicular to the stretching direction after cessation of flow for long, well-entangled polyisoprene chains of $Z=58$. In view of these contradictory results we present a study of the conformational relaxation behavior of polymer melts right after a large step elongation for different numbers of entanglements per chain ranging from about $Z=18$ to $Z=72$. By comparing chain conformations and an expansion of small angle scattering patterns [36, 37] in spherical harmonics [46] we demonstrate how the overall scattering patterns infer the internal structure of the chain conformations.

We have performed extensive molecular dynamics (MD) simulations of strongly deformed polymer melts [34] using the ESPResSo++ package [47]. Starting from fully equilibrated melts of highly entangled beadspring chains with a weak bond bending constant of $k_{\theta}=$ 
(a)

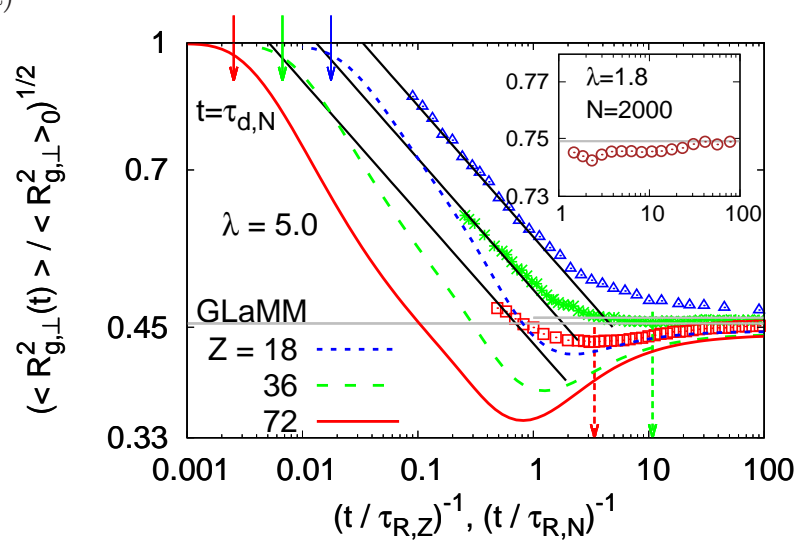

(b)

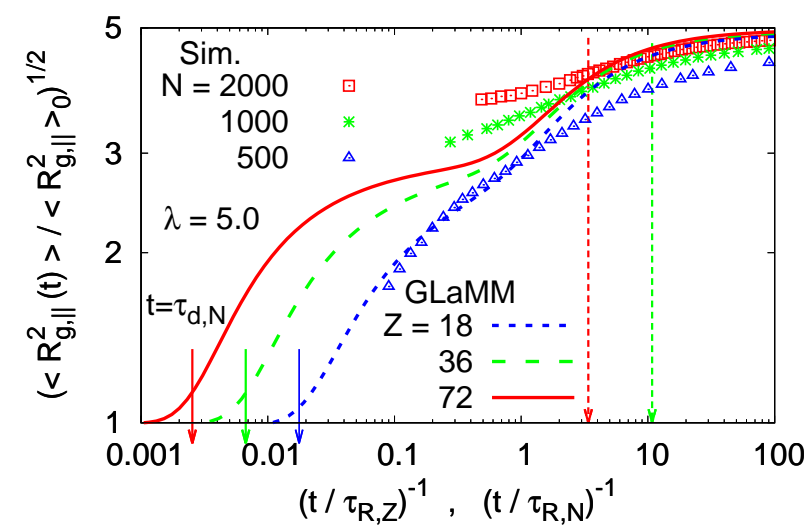

FIG. 1. Log-log plot of the rescaled root mean square radius of gyration in the direction perpendicular and parallel to the stretching direction, $\left(\left\langle R_{g, \perp}^{2}(t)\right\rangle /\left\langle R_{g, \perp}^{2}\right\rangle_{0}\right)^{1 / 2}$ (a) and $\left(\left\langle R_{g,||}^{2}(t)\right\rangle /\left\langle R_{g,||}^{2}\right\rangle_{0}\right)^{1 / 2}$ (b), respectively, plotted versus inverse rescaled time, $\left(t / \tau_{R, N}\right)^{-1}$, for $N=500,1000$, and 2000, as indicated. The corresponding disentanglement times $t=\tau_{d, N}$ are pointed by solid arrows near one on the $y$ axis. Theoretical predictions from the GLaMM model [35] versus $\left(t / \tau_{R, Z}\right)^{-1}$ are shown for comparison. Minimum values of $\left\langle R_{g, \perp}^{2}(t)\right\rangle /\left\langle R_{g, \perp}^{2}\right\rangle_{0}$ are marked by dashed arrows, which also indicate the onset of relaxation delay in (b). The horizontal line in (a) gives $\left(\left\langle R_{g, \perp}^{2}(t)\right\rangle /\left\langle R_{g, \perp}^{2}\right\rangle_{0}\right)^{1 / 2}$ right after elongation for $N=1000$ and 2000 . Straight lines $\left(a_{g} x^{-b_{g}}\right)$ indicate best fits to our simulation data for $\left(\tau_{R, N} / t\right)<1.0$ (cf. text). For comparison data for $N=2000$ at $\lambda=1.8$ are shown in the inset of (a).

$1.5 \epsilon[9,17,18,39,48]$ chains behave as ideal chains above the Kuhn length corresponding to $2.8(1) \ell_{b}, \ell_{b} \approx 0.964 \sigma$ being the bond length. Isothermal MD simulations at temperature $T=1 \epsilon / k_{B}$ have been performed (for details see the Supplemental Material in Ref. 34]). LennardJones energy, time, and length units denoted by $\epsilon, \tau$, and $\sigma$, respectively, are used throughout this work. For these parameters the entanglement length corresponds to $N_{e}=28$ monomers [17, 39]. We apply an isochoric and uniaxial elongation along the $x$ direction with a deformation rate $\dot{\varepsilon}=77 \tau_{R, N}^{-1}=\left(77 / Z^{2}\right) \tau_{e}^{-1}$, i.e. $\tau_{R, N}^{-1}<\dot{\varepsilon}<\tau_{e}^{-1}$, up to a total strain of $\lambda=L_{x} / L_{0}=5$. Here $\tau_{R, N}=\tau_{0} N^{2}$ with $\tau_{0}=2.89 \tau$ and $\tau_{e}=\tau_{0} N_{e}^{2}$ are Rouse times of a chain of length $N$ and of an entanglement length $N_{e}$, respectively. This is the relevant nonlinear viscoelastic regime, where a delicate interplay between deformation rate and internal conformational relaxation plays a crucial role [24, 32, 33, 35]. For times up to about the Rouse time of the chains we have seen that the relaxation along the tube is by no means homogeneous; i.e., the primitive paths [49 51] exhibit long-lived clustering of topological constraints in the deformed state, leading to significantly delayed relaxation [34]. This is not accounted for by any of the current theoretical concepts. Here, however, we focus on the initial relaxation of experimentally more directly accessible global conformational properties of deformed polymer melts, where observed deviations from the GLaMM model 35 have been taken to question the validity of the tube concept as a whole [36, 37, 41].

Subject to uniaxial elongation the average conformation of single chains in a melt exhibits axial symmetry along the stretching direction ( $x$ axis). Therefore, the mean square radius of gyration which describes the chain conformations should be decomposed into two components parallel and perpendicular to the stretching direction, i.e. $\left\langle R_{g}^{2}\right\rangle=\left\langle R_{g, \|}^{2}\right\rangle+\left\langle R_{g, \perp}^{2}\right\rangle$, and $\left\langle R_{g, \|}^{2}\right\rangle=\left\langle R_{g, \perp}^{2}\right\rangle / 2$ in equilibrium.

Based on the tube model one would expect an overdamped initial retraction process in both directions parallel and perpendicular to the $x$ axis [5, 35]. While this is obvious for the extension parallel to the stretching direction, this effect is expected to be much weaker for the perpendicular one, as it eventually has to turn and increase towards the equilibrium value. Time evolution of the rescaled two components of radius of gyration, $\left(\left\langle R_{g, \perp}^{2}\right\rangle /\left\langle R_{g, \perp}^{2}\right\rangle_{0}\right)^{1 / 2}$ and $\left(\left\langle R_{g, \|}^{2}\right\rangle /\left\langle R_{g, \|}^{2}\right\rangle_{0}\right)^{1 / 2}$, for single chains of sizes $N=500,1000$, and $2000(Z \approx 18,36$, and 72) in melts during relaxation are shown in Fig. 1 and compared to the GLaMM model 35, 52] (see Supplemental Material [53]). The symbols $\langle\cdots\rangle$ and $\langle\cdots\rangle_{0}$ stand for the average over $n_{c}=1000$ chains in deformed and unperturbed (i.e., fully equilibrated) polymer melts, respectively. The parameters $c_{\nu}=0.1$ and $R_{s}=2.0$ are set to the same values as they were tested in the GLaMM model [35]. Except for $\left(\left\langle R_{g, \perp}^{2}\right\rangle /\left\langle R_{g, \perp}^{2}\right\rangle_{0}\right)^{1 / 2}$ of the shortest chains of $N=500$ (i.e., $Z \approx 18$ ), we see that both components of $R_{g}$ for deformed polymer melts initially decrease with increasing relaxation time. Evidently, our results qualitatively capture the signature of the initial chain retraction mechanism [5, 35] right after a large step elongation.

Since the chains finally must relax to their equilib- 
(a)

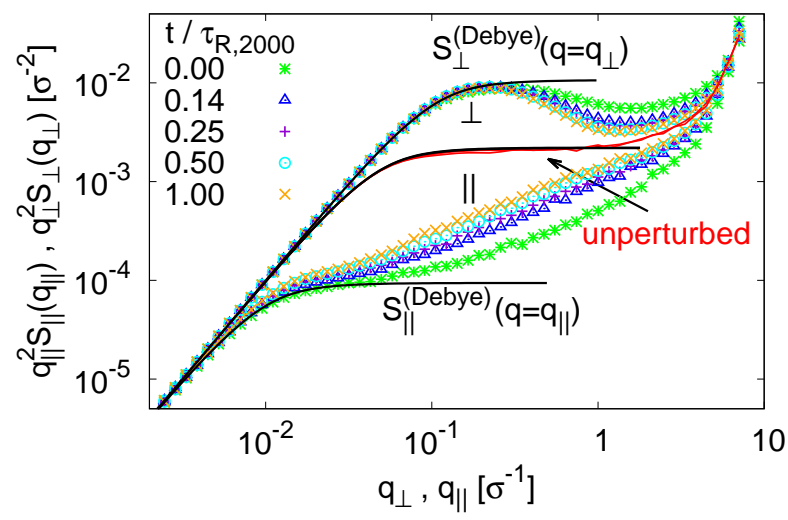

(b)

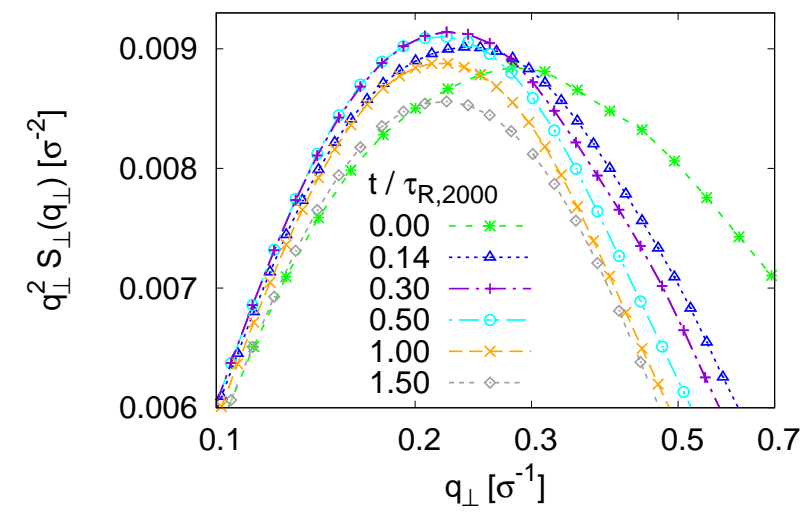

FIG. 2. Kratky-plot of the two components of the single chain structure factor parallel and perpendicular to the stretching direction, $q_{\|}^{2} S_{\|}\left(q_{\|}\right)$and $q_{\perp}^{2} S_{\perp}\left(q_{\perp}\right)$, respectively (a), and $q_{\perp}^{2} S_{\perp}\left(q_{\perp}\right)$ for $0.1 \sigma^{-1}<q_{\perp}<0.7 \sigma^{-1}$ (b). Data are for chains of size $N=2000$. Several values of the relaxation time $t / \tau_{R, N}$ are shown, as indicated. Data for the unperturbed polymer melt (red curve) and the decomposed Debye functions [54, 55]: $S_{\alpha}^{(\text {Debye) }}\left(q=q_{\alpha}\right)=2\left[\exp \left(-X_{\alpha}\right)-1+X_{\alpha}\right] / X_{\alpha}^{2}$ with $X_{\alpha=\|}=3 q_{\| \mid}^{2}\left\langle R_{g, \|}^{2}\right\rangle$ and $X_{\alpha=\perp}=3 q_{\perp}^{2}\left\langle R_{\perp}^{2}\right\rangle / 2$ are also shown in (a) for comparison. Note that $S_{\|}^{(\text {Debye })}(q)=S_{\perp}^{\text {(Debye) }}(q)$ for unperturbed polymer melts.

rium conformation, $\left(\left\langle R_{g, \perp}^{2}\right\rangle /\left\langle R_{g, \perp}^{2}\right\rangle_{0}\right)^{1 / 2}$ will go through a minimum until it increases towards unity. In Fig. 1(a) we see that $\left(\left\langle R_{g, \perp}^{2}\right\rangle /\left\langle R_{g, \perp}^{2}\right\rangle_{0}\right)^{1 / 2}$ first decreases, reaches a minimum at $t / \tau_{R, 1000}=0.09$ for $N=1000$ and $t / \tau_{R, 2000}=0.30$ for $N=2000$, and then turns around and gradually increases. With increasing $Z$ the minimum becomes more pronounced and is shifted to later times, however still below $\tau_{R, N}$. The GLaMM model predicts a minimum at $t \approx \tau_{R, Z}$ and a significantly stronger signature of retraction for the same values of $Z$, thus only qualitatively agreeing to our data. NNote that our data for $N=2000$ and $\lambda=1.8$ shown in Fig. I(a) (inset) indicate the signature becomes much weaker with decreasing $\lambda$. From that it is not surprising that the minimum in $\left\langle R_{g, \perp}^{2}\right\rangle$ has not been observed in Ref [37]]. It is tempting to extrapolate the data to $\left(\left\langle R_{g, \perp}^{2}\right\rangle /\left\langle R_{g, \perp}^{2}\right\rangle_{0}\right)^{1 / 2}=1$ by a fitting function $f(x)=a_{g} x^{-b_{g}}$ for $t>\tau_{R, N}$. For $N=500$ we estimate $b_{g}=0.16$ and $a_{g}=0.58$. Assuming the same power law for $N=1000,2000$, because all systems are deep in the entangled regime, we arrive at $a_{g}=0.50$ and 0.43 , for $N=1000,2000$. By that we obtain equilibration time estimates of $t_{\mathrm{eq}, N}[=$ $\left.\left(1 / a_{g}\right)^{1 / b_{g}} \tau_{R, N}\right]=30 \tau_{R, 500}, 76 \tau_{R, 1000}$, and $195 \tau_{R, 2000}$, close to $\tau_{d, N} / 2, \tau_{d, N}=\left(N / N_{e}\right)^{1.4} \tau_{R, N}$. For the GLaMM model, one obtains $t_{\mathrm{eq}, Z}=50 \tau_{R, Z=18}, 148 \tau_{R, Z=36}$, and $363 \tau_{R, Z=72}$ with the parameters $b_{g}=0.21$ and $a_{g}=0.44$, 0.35 , and 0.29 for $Z=18,36$, and 72 , respectively, based on $\tau_{d, Z}=Z^{1.4} \tau_{R, Z}$. The above assumes an unperturbed relaxation until isotropic chain conformations are reached. Though intuitive, this most probably cannot be the case, as indicated by the data for $\left\langle R_{g, \|}^{2}\right\rangle$, as well as by previous primitive path analysis [34]. $\left(\left\langle R_{g, \|}^{2}\right\rangle /\left\langle R_{g, \|}^{2}\right\rangle_{0}\right)^{1 / 2}$ decreases monotonically with time $t$ while the relaxation rate still becomes smaller with time $(N=1000,2000)$. Eventually we observe the signature of an intermediate plateau well above and significantly earlier than the regime predicted by GLaMM, pointing towards a significantly delayed conformational relaxation. This relaxation retardation of the deformed chains has been attributed to an inhomogeneous distribution of entanglement points along the primitive paths 34], not accounted for in current theoretical models. A similar delay has been observed in the context of rheological experiments of very long, highly entangled polymer chains by several authors 56 58]. The GLaMM model predicts the equilibrium melt disentanglement time of the chain to be the longest relaxation time.

Experimentally scattering functions are more easily accessible. The normalized single chain structure factor $S(\mathbf{q})$ will easily detect any anisotropy after deformation. As for $R_{g}$ we distinguish $S_{\|}\left(q_{\|}=q_{x}\right)$ where the wave vector $\mathbf{q}$ is oriented in the $x$ direction parallel to the stretching direction, and $S_{\perp}\left[q_{\perp}=\left(q_{y}^{2}+q_{z}^{2}\right)^{1 / 2}\right]$. Note that here we discuss the static structure factor for deformed polymers in melts at certain selected relaxation times. In Fig. 2 we present the two components $S_{\|}\left(q_{\|}\right)$ and $S_{\perp}\left(q_{\perp}\right)$ for $N=2000$ in deformed $(\lambda=5)$ melts. After a large step elongation, $S_{\|}\left(q_{\|}\right)$and $S_{\perp}\left(q_{\perp}\right)$ strongly deviate from ideality. In the Guinier regime, $q<2 \pi / R_{g}$, our data are very well described by the decomposed Debye function, as indicated. With increasing relaxation time, the range over which the ideal behavior holds slowly extends, however, it remains still far from that of ideal chains. As expected from $R_{g}$ the chain retraction as ob- 
(a)

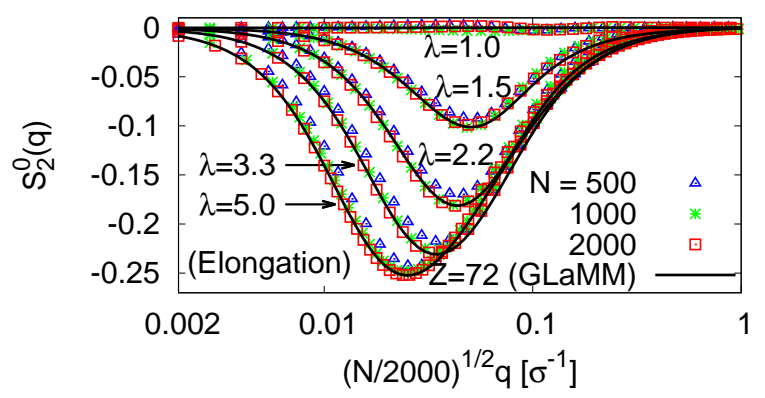

(b)

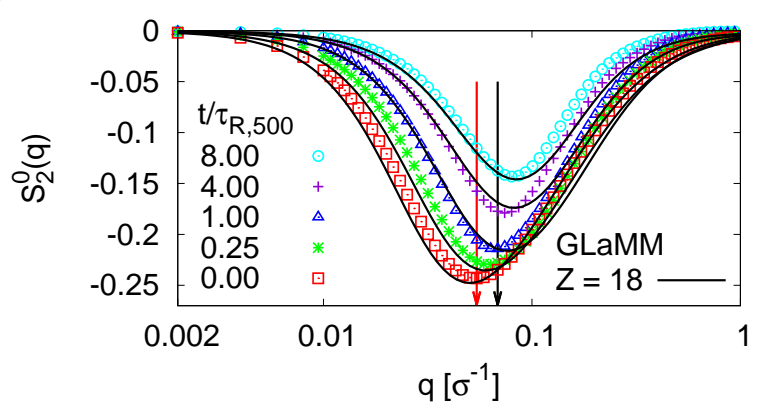

(c)

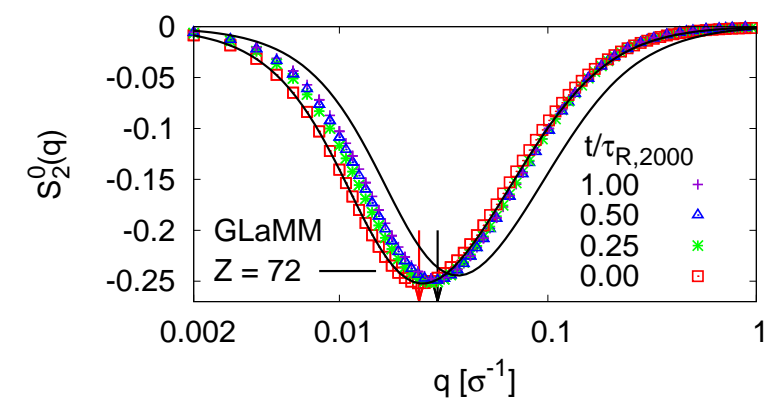

FIG. 3. Leading anisotropic term of the single chain structure factor, $S_{2}^{0}(q)$, plotted versus $q$ for elongated polymer melts (a) and upon subsequent relaxation for chain sizes $N=500$ (b), and 2000 (c). In (a) five values of stretching ratio $\lambda$, and three different chain sizes $N$ are chosen, as indicated. In (b)(c) data are for several subsequent relaxation times $t / \tau_{R, N}$ after stretching, as indicated. The predicted results from the GLaMM model are shown by black curves for all cases in (b), but only for $t / \tau_{R, 2000}=0$ and 1 in (c). Note that for the GLaMM model $q$ here is rescaled to $0.62 q N_{e}^{-1 / 2}$ such that simulation data and theoretical predictions are coincidental in (a).

served for $N=2000$, clearly shows up in the Kratky plot of the structure factor $S_{\perp}\left(q_{\perp}\right)$ [Fig. 2(b)]. The peak height first increases for times up to $t / \tau_{R, 2000}=0.3$, the time where $\left\langle R_{g, \perp}^{2}\right\rangle$ reaches a minimum (see Fig. 10). Then it decreases as $\left\langle R_{\perp}^{2}\right\rangle$ turns to increase. This reduction for $t / \tau_{R, 2000}>0.3$, reveals a reduction of the anisotropy.

To compare this to theoretical predictions we follow previous work of Refs. [36, 37] and employ an expansion with respect to spherical harmonics to the single chain structure factor $S(\mathbf{q})$. This should reveal the relationship between anisotropic chain structure and chain retraction for the leading anisotropic term. To take into account axial symmetry, we choose the polar angle $\theta$ to be the angle between $\mathbf{q}$ and the $x$ axis. Then the structure factor is independent of the azimuthal angle $\phi$, implying that the expansion with respect to the spherical harmonics $Y_{\ell}^{m}(\theta, \phi)$ exhibits only terms with $m=0$, $Y_{\ell}^{0}(\theta)$. In practice, we simply set $\phi=0$ and thus obtain $S\left(q_{x}=q \cos \theta, q_{z}=q \sin \theta\right)=\sum_{\ell=0,2,4, \ldots} S_{\ell}^{0}(q) Y_{\ell}^{0}(\theta)$, where odd $\ell$ values do not occur for reasons of mirror symmetry. Focusing on the leading order anisotropy, we present in Fig. 3 the coefficient $S_{2}^{0}(q)$, for polymer melts of our three different chain sizes within the elongation process at five selected strain values $\lambda$, and during the relaxation process at fixed $\lambda=5.0$. Since polymer chains deform affinely, and $q \propto 1 /\left\langle R_{g}^{2}\right\rangle^{1 / 2} \propto 1 / N^{1 / 2}$, we rescale $q$ to $(N / 2000)^{1 / 2} q$ in Fig. 3)(a). As expected, we observe a nice data collapse for chains of different $N$. With increasing $\lambda$, the anisotropy of deformed polymer chains in a melt is enhanced. The differences between the gyration radii along the $x$ and $z$ axes become more pronounced, resulting in a horizontal shift of $S_{2}^{0}(q)$ to smaller values of $q$. Meanwhile, the orientation anisotropy becomes stronger, i.e. the minimum of $S_{2}^{0}(q)$ becomes deeper. So far the agreement between the GLaMM model and the simulation is excellent.

As the deformed chains start to relax, the situation changes. For all cases [Fig. 3(b) and 3(c)], we indeed see a horizontal shift of $S_{2}^{0}(q)$ to larger values of $q$ due to the shrinkage of chains within the initial relaxation up to about the Rouse time. The minimum of $S_{2}^{0}(q)$ becomes more shallow, depending on the number of entanglements $Z$. For better illustration, the minima at $t / \tau_{R, N} \approx 0$ and 1.0 are indicated by arrows. As observed directly by analyzing $R_{g}$ the GLaMM model seems to reproduce this relaxation better for small $Z$, indicating significant deviations from the GLaMM relaxation mechanisms with increasing chain length. Results of the higher order terms $S_{4}^{0}(q)$ and $S_{6}^{0}(q)$ are shown in Supplemental Material [53].

In summary, both results of the radius of gyration and the one-dimensional structure factor of deformed melts indicate that chain retraction in all directions sets in during initial relaxation before reaching the Rouse time. We find that the signature becomes more pronounced with an increasing number of entanglements $Z$ as predicted by the GLaMM model. Such an effect was not observed in Refs. 36, 37]. Our data indicate that there the number of entanglements $Z$ is not big enough and/or the applied strain is not large enough; i.e., the stretch ratio $\lambda=1.8$ is too small. We have also shown that during the relaxation process up to the Rouse time, the leading anisotropic term of the single chain structure factor follows a similar pattern as predicted by the GLaMM tube model. Beyond the initial agreement with the GLaMM model at 
short times significant deviations have been observed for larger times. This relaxation retardation needs further investigation, as it points to different, not yet understood relaxation pathways in the nonlinear viscoelastic regime of highly entangled polymer melts.

\section{ACKNOWLEDGEMENT}

We are grateful to B. Dünweg for a critical reading of the manuscript. H.-P. H. thanks R. Graham for helpful discussions on the GLaMM tube model. This work has been supported by the European Research Council under the European Union's Seventh Framework Programme (FP7/2007-2013)/ERC Grant Agreement No. 340906MOLPROCOMP. We gratefully acknowledge the computing time granted by the John von Neumann Institute for Computing (NIC) and provided on the supercomputer JUROPA at Jülich Supercomputing Centre (JSC), and the Max Planck Computing and Data Facility (MPCDF).

* hsu@mpip-mainz.mpg.de

kremer@mpip-mainz.mpg.de

[1] P. G. de Gennes, Scaling Concepts in polymer physics (Cornell University Press: Itharca, New York, 1979).

[2] M. Doi, J. Polym. Sci. Polym. Phys. Ed. 18, 1005 (1980).

[3] M. Doi, J. Polym. Sci. Polym. Phys. Ed. 19, 229 (1981).

[4] M. Doi, J. Polym. Sci. Polym. Phys. Ed. 21, 667 (1983).

[5] M. Doi and S. Edwards, The theory of polymer dynamics (Oxford University Press: New York, 1986).

[6] M. Rubinstein and R. H. Colby, Polymer Physics (Oxford University Press, Oxford, 2003).

[7] S. F. Edwards, Proc. Phys. Soc. 91, 513 (1967).

[8] K. Kremer, G. S. Grest, and I. Carmesin, Phys. Rev. Lett. 61, 566 (1988).

[9] K. Kremer and G. S. Grest, J. Chem. Phys. 92, 5057 (1990).

[10] W. Paul, K. Binder, D. W. Heermann, and K. Kremer, J. Chem. Phys. 95, 7726 (1991).

[11] J. Wittmer, W. Paul, and K. Binder, Macromolecules 25, 7211 (1992).

[12] K. Kremer and G. S. Grest, J. Chem. Soc. Faraday Trans 88, 1707 (1992).

[13] A. Kopf, B. Dünweg, and W. Paul, J. Chem. Phys. 107, 6945 (1997).

[14] M. Pütz, K. Kremer, and G. S. Grest, Europhys. Lett. 49, 735 (2000).

[15] V. A. Harmandaris, V. G. Mavrantzas, D. N. Theodorou, M. Kröger, J. Ramirez, H. C. Öttinger, and D. Vlassopoulos, Macromolecules 36, 1376 (2003).

[16] G. Tsolou, V. G. Mavrantzas, and D. N. Theodorou, Macromolecules 38, 1478 (2005).

[17] H.-P. Hsu and K. Kremer, J. Chem. Phys. 144, 154907 (2016).

[18] H.-P. Hsu and K. Kremer, Eur. Phys. J. Special Topics 226, 693 (2017).

[19] L. J. Fetters, D. J. Lohse, D. Richter, T. A. Witten, and A. Zirkel, Macromolecules 27, 4639 (1994).
[20] A. Wischnewski and D. Richter, in Soft Matter, Vol. 1:Polymer melts and mixtures, edited by G. Gompper and M. Schick (Wiley-VCH, Weinheim, 2006) Chap. 1, pp. 17-85.

[21] W. W. Graessley, Polymeric liquids \& networks: structure and properties (Garland Science, London and New York, 2008).

[22] A. Herrmann, B. Kresse, M. Wohlfahrt, I. Bauer, A. F. Privalov, D. Kruk, N. Fatkullin, F. Fujara, and E. A. Rössler, Macromolecules 45, 6516 (2012).

[23] T. P. Lodge, Phys. Rev. Lett. 83, 3218 (1999).

[24] A. E. Likhtman and T. C. B. McLeish, Macromolecules 35, 6332 (2002).

[25] M. Abdel-Goad, W. Pyckhout-Hintzen, S. Kahle, J. Allgaier, D. Richter, and L. J. Fetters, Macromolecules 37, 8135 (2004).

[26] A. E. Likhtman and M. Ponmurugan, Macromolecules 47, 1470 (2014).

[27] F. Furtado, J. Damron, M.-L. Trutschel, C. Franz, K. Schröter, R. C. Ball, K. Saalwächter, and D. Panja, Macromolecules 47, 256 (2014).

[28] J. Klein, Macromolecules 11, 852 (1978).

[29] M. Daoud and P. G. de Gennes, J. Polym. Sci. Polym. Phys. Ed. 17, 1971 (1979).

[30] M. Rubinstein and R. H. Colby, J. Chem. Phys. 89, 5291 (1988).

[31] S. T. Milner, T. C. B. McLeish, and A. E. Likthman, J. Rheol. 45, 539 (2001).

[32] T. C. B. McLeish, Adv. Phys. 51, 1379 (2002).

[33] T. C. B. McLeish, Rheol. Rev. , 197 (2003).

[34] H.-P. Hsu and K. Kremer, ACS Macro Lett. 7, 107 (2018).

[35] R. S. Graham, A. E. Likhtman, T. C. B. McLeish, and S. T. Milner, J. Rheol. 47, 1171 (2003).

[36] Z. Wang, C. N. Lam, W.-R. Chen, W. Wang, J. Liu, Y. Liu, L. Porcar, C. B. Stanley, Z. Zhao, K. Hong, and Y. Wang, Phys. Rev. X 7, 031003 (2017).

[37] W.-S. Xu, J.-M. Y. Carrillo, C. N. Lam, B. G. Sumpter, and Y. Wang, ACS Macro Lett. 7, 190 (2018).

[38] R. S. Hoy, K. Foteinopoulou, and M. Kröger, Phys. Rev. E 80, 031803 (2009).

[39] L. A. Moreira, G. Zhang, F. Müller, T. Stuehn, and K. Kremer, Macromol. Theor. Simul. 24, 419 (2015).

[40] J. Bent, L. R. Hutchings, R. W. Richards, T. G. R. Spares, P. D. Coates, I. Grillo, G. Harlen, D. J. Read, R. S. Graham, A. E. Likhtman, D. J. Groves, T. M. Nicholson, and T. C. B. McLeish, Science 301, 1691 (2003).

[41] A. Blanchard, R. S. Graham, M. Heinrich, W. PyckhoutHintzen, D. Richter, A. E. Likhtman, T. C. B. McLeish, D. J. Read, E. Straube, and J. Kohlbrecher, Phys. Rev. Lett. 95, 166001 (2005).

[42] R. S. Graham, J. Bent, L. R. Hutchings, R. W. Richards, D. J. Groves, J. Embery, T. M. Nicholson, T. C. B. McLeish, A. E. Likhtman, O. G. Harlen, D. J. Read, T. Gough, R. Spares, P. D. Coates, and I. Grillo, Macromolecules 39, 2700 (2006).

[43] R. Muller and C. Picot, Makromol. Chem. Macromol. Symp. 56, 107 (1992).

[44] R. Muller, thése d'état, Université Louis Pasteur de Strasbourg (1988).

[45] M. Kröger, C. Luap, and R. Muller, Macromolecules 30, 526 (1997). 
[46] P. Lindner and S. Hess, Physica B 156 \& 157, 512 (1989).

[47] J. D. Halverson, T. Brandes, O. Lenz, A. Arnold, S. Bevc, V. Starchenko, K. Kremer, T. Stuehn, and D. Reith, Comput. Phys. Commun. 184, 1129 (2013).

[48] G. Zhang, L. A. Moreira, T. Stuehn, K. C. Daoulas, and K. Kremer, ACS Macro Lett. 3, 198 (2014).

[49] R. Everaers, S. K. Sukumaran, G. S. Grest, C. Svaneborg, A. Sivasubramanian, and K. Kremer, Science 303, 823 (2004).

[50] S. K. Sukumaran, G. S. Grest, K. Kremer, and R. Everaers, J. Polym. Sci. B 43, 917 (2005).

[51] R. Everaers, Phys. Rev. E 86, 022801 (2012).

[52] R. S. Graham, Macromolecules 46, 9849 (2013).

[53] See Supplemental Material at http://link.aps.org /supplemental/10.1103/PhysRevLett.121.167801 for the solution of the GLaMM model and the detailed comparison between the theoretical predictions and simulation results.

[54] H. Benoit, R. Duplessix, R. Ober, M. Daoud, J. P. Cotton, B. Farnoux, and G. Jannink, Macromolecules 8, 451 (1975).

[55] H.-P. Hsu, W. Paul, and K. Binder, J. Chem. Phys. 137, 174902 (2012).

[56] L. A. Archer, Y. L. Chen, and R. G. Larson, J. Rheol. 39, 519 (1995).

[57] L. A. Archer, J. Sanchez-Reyes, and Juliani, Macromolecules 35, 10216 (2002).

[58] D. C. Venerus and R. Nair, J. Rheol. 50, 59 (2006). 